\section{Salud mental y literaturas no realistas: aportes de la extensión a la investigación}

\author{
Lucas Cabrera Rodriguez \\ lucas_rodriguez13@hotmail.com \\ María Estefanía Pagano Artigas \\ estefania.pagano@gmail.com
}

\author{
Andrés Vázquez \\ andres.vazquez.l@gmail.com \\ Universidad de la República, \\ Uruguay
}

Cultura(s) en clave de extensión universitaria / Intervenciones
RECEPCIÓN: 24/06/18

ACEPTACIÓN FINAL: 30/05/19

\section{Resumen}

El presente artículo busca reflexionar sobre los aportes de la extensión a la investigación mediante la articulación de la salud mental y las literaturas no realistas a partir del Espacio de Formación Integral (dispositivo que involucra actividades de extensión, enseñanza e investigación): "Taller abierto de lectura, interpretación y creación en torno a literaturas no realistas, insólitas y fantásticas en el Hospital Vilardebó", de la Facultad de Humanidades y Ciencias de la Educación de la Universidad de la República. Se intenta reflejar cómo dicha experiencia extensionista que promueve integralidad, aporta, contribuye y colabora a repensar la investigación "tradicional" que se percibe del Grupo de Investigación Raros y Fantásticos en la Literatura Uruguaya. Teoría, Crítica e Historia (1963-2004) y así posibilitar una nueva forma de investigar: acompañada de la extensión.

Palabras clave: extensión universitaria Espacio de Formación Integral, literatura no realista, salud mental.
Mental health and non-realistic fiction: contribution of extension practices to research

\section{Abstract}

This article aims to reflect on how extension practices at University level may contribute to literature theory research. Within the Division of Integral Education (scholar device that combines extension practices, teaching and researching) of Facultad de Humanidades y Ciencias de la Educación (Universidad de la República), and under the name of "Open Workshop of lecture, creation and interpretation toward non-realistic fiction at Viladrebó Hospital", some extension experiences in the field of Mental Health were carried on by Humanities scholars. Some interesting considerations rewarding the interaction between mental health and literature emerged throughout that experience; to present those thoughts is intended here.

Keywords: extension practices, Integral education, non-realistic fiction, mental health.
Saúde mental e literaturas irrealistas: contribuições da extensão à pesquisa

\section{Resumo}

O presente texto procura refletir sobre as contribuições da extensão à pesquisa através da articulação da Saúde Mental e literaturas não realistas a partir do Espaço de Formação Integral (dispositivo que envolve atividades de extensão, ensino e pesquisa): "Oficina de leitura, interpretação e criação aberta em torno de literaturas não realistas, estranhas e fantásticas no Hospital Vilardebó" da Faculdade de Ciências Humanas e da Educação da Universidade da República. Procuramos refletir como essa experiência de extensão que promove a integralidade contribui e colabora para repensar a pesquisa "tradicional" que é percebida pelo Grupo de Pesquisa Raros e Fantásticos da Literatura Uruguaia. Teoria, Crítica e História (1963-2004) e assim possibilitar uma nova forma de pesquisa: acompanhada de extensão.

Palavras-chave: extensão universitária, Espaço de Formação Integral, literatura não realista, Saúde Mental.

Para citación de este artículo: Cabrera Rodriguez, L.P.; Pagano Artigas, M.E., y Vázquez, A. (2019). Salud mental y literaturas no realistas: aportes de la extensión a la investigación. +E: Revista de Extensión Universitaria, 9(10), 137-150. doi: 10.14409/extension.v9i10.Ene-Jun.8297. 
"El Departamento de Extensión Universitaria está al servicio del pueblo para todo lo que contribuya a su enaltecimiento. Marta Elena Samatán, 1958." (Scarciófolo, 2017:78)

\section{Introducción}

Con este artículo pretendemos, en primer lugar, compartir una experiencia de extensión universitaria desarrollada por el Departamento de Literatura Uruguaya y Latinoamericana de la Facultad de Humanidades y Ciencias de la Educación (FHCE) de la Universidad de la República (UdelaR) en el único hospital psiquiátrico público del Uruguay llamado Hospital Vilardebó. Dicha experiencia desarrollada bajo el dispositivo de Espacio de Formación Integral (EFI) se llama "Taller abierto de lectura, interpretación y creación en torno a literaturas no realistas, insólitas y fantásticas en el Hospital Vilardebó". A partir de la presentación de la experiencia de extensión universitaria, buscamos reflexionar cómo se relacionan las funciones universitarias, es decir, cuáles son los aportes de esta experiencia a las otras funciones universitarias, en particular a la investigación, dando cuenta del modo en que la extensión impacta en la investigación y en qué ésta última es modificada. Asimismo, aspiramos a reflexionar sobre cómo se desarrolla la integralidad, no solo entre las funciones, sino entre la diversidad de estudiantes de distintas carreras o áreas que participan en ella, cuál es el papel del estudiante, cómo transcurre y cuáles conocimientos y saberes se ponen en juego.

Vale resaltar que esta experiencia se desarrolla en el marco de la nueva Ley de Salud Mental que en Uruguay se aprobó en 2017. La perspectiva de trabajo del EFI es desde los derechos humanos. No se trata de una experiencia que responda a un modelo biologicista.

El artículo hará un recorrido sobre la concepción de la extensión en UdelaR y los EFI, relatará el origen, la creación y la descripción de esta experiencia "Un EFI en un manicomio", que se aproxima al porqué de un EFI en un manicomio en el marco de la nueva Ley de Salud Mental, y analizará el impacto de la experiencia en las otras dos funciones universitarias, en los estudiantes, en el hospital y en la concepción de investigación literaria.

\section{Historizando nuestra extensión}

Las conmemoraciones de las fechas exactas son las que colaboran en cuanto a detenernos y repensar lo que intenta estar siempre presente en nuestras prácticas universitarias. En 2018 se cumplieron los 100 años que nos separan del momento en que el movimiento estudiantil de Córdoba (Argentina) hacía historia. El 21 de junio de 1918 se lanzaba el Manifiesto Liminar. A partir de la Reforma Universitaria, la extensión pasó a ser una de las tres funciones sustantivas de la universidad; las otras, enseñanza e investigación. Al referirnos a la extensión universitaria, consideramos: "la relación interactiva que la universidad plantea con su medio en las dimensiones sociales, culturales y productivas, a partir de la circulación del conocimiento y en la búsqueda de la democratización y apropiación social del mismo" (Menéndez, 2017:29) y encontramos las siguientes dimensiones: académico-institucional, comunicacional en términos dialógicos, social en términos de transformación, pedagógica, política (Menéndez, 2017). 
En Uruguay, la "Segunda Reforma Universitaria" (Arocena, 2011), desarrollada entre 2006 y 2014, fue la que propició, entre otras cuestiones, la curricularización de la extensión. Con esto, la extensión pasa a ser una actividad del estudiante comprometido para integrarse a la vida académica junto a la enseñanza y la investigación. Estas tres funciones universitarias comenzaron a verse como un todo y no como compartimentos estanco. De este modo, la universidad funciona desde la integralidad. $Y$ entendemos por integralidad, desde la perspectiva extensionista, la articulación de las tres funciones universitarias, es decir, docencia, extensión e investigación, que apuestan a la formación crítica y humanista de los estudiantes, de las distintas disciplinas, fomentando así la interdisciplina, del diálogo de saberes entre diversos actores sociales y la articulación en la construcción intersectorial e interinstitucional de propuestas que resuelvan problemáticas concretas y que tengan un espíritu transformador (Tommasino, Cano, Castro, Santos y Stevenazzi, 2010; Cano, 2015).

Entre los dispositivos creados para llevar a cabo la integralidad de las tres funciones universitarias están los EFI. No hay una estructura rígida acerca de lo que son. Pueden estar compuestos por distintas experiencias educativas que tengan como eje central el diálogo con la sociedad no solo desde la extensión sino también desde la enseñanza y la investigación. Pueden ser un conjunto de prácticas, cursos, talleres, proyectos, pasantías. En general, los EFI son organizados por docentes universitarios de distintas facultades y pensados para estudiantes de distintas áreas del saber, ya que es la interdisciplina un aspecto fundamental del EFI. Estos "tienen como propósitos articular las funciones universitarias, promover la autonomía del estudiante, el pensamiento crítico propositivo, así como fomentar el trabajo en grupo desde una perspectiva interdisciplinaria" (Castillo, Correa, Parentelli, y Romero, 2017:157).

Sin embargo, en la actualidad, la extensión universitaria (y no solo esta) sufre un fuerte intento de recorte y de regresión. Son tiempos donde la contrarreforma neoliberal subsume a las universidades a las demandas del mercado, neutraliza las organizaciones docentes y dispersa los movimientos estudiantiles con la diversificación segmentada de la oferta educativa (Cano, 2017). Debido a esta coyuntura, se nos hace imprescindible recurrir a nuestra herencia latinoamericana y reconstruir el pasado en nuestro presente. Tal como expresa Scarciófolo: "Preservar las fuentes del pasado nos ofrece la posibilidad de poder reconstruirlo una y otra vez" (2018:s/p).

De esta manera, nuestras intervenciones en la intersección entre salud mental y literaturas no realistas en el Hospital Vilardebó buscan crear un espacio de formación integral con la finalidad de resistir a la coyuntura neoliberal mencionada más arriba que afecta tanto a la universidad como a las prácticas de intervención en salud mental. En la búsqueda de propiciar la organización de un espacio de escucha afectiva, horizontalidad y respeto, lo que implica un acercamiento a una población en los márgenes de la sociedad, surge una experiencia de aprendizaje de gran valor y una nueva forma de estudiar literaturas no realistas que transforman las lógicas de investigación y de enseñanza exclusivamente académicas.

Intentamos mantener y promover, por un lado, el legado de Córdoba, y por el otro, lo que se desarrolló en UdelaR en el período que refiere este artículo, en tanto que, a continuación, desarrollaremos cómo surgió nuestro EFI. 


\section{De la investigación a la extensión: creación y descripción del EFI}

En el año 2014, en el Departamento de Literaturas Uruguaya y Latinoamericana de FHCE de la UdelaR, se originó el "Grupo de investigación Raros y Fantásticos en la Literatura Uruguaya. Teoría, Crítica e Historia (1963-2004)". En el mismo nos dedicamos a estudiar obras de autores uruguayos que se distancian de lo mimético, de aquella literatura realista propia del Uruguay del siglo XX. En otros términos, la actividad radica en el estudio críticoliterario de las características poéticas de autores uruguayos que están en los márgenes del canon literario y de la sociedad, donde se hace patente en su narrativa la recurrencia del tema de lo otro y el carácter alterno más que de los protagonistas, del modo en que ellos viven -o narran - sus experiencias.

El tema de lo otro y el carácter alterno son propios de estas literaturas raras, insólitas, extrañas, fantásticas, no realistas. Se trata de la presencia de algo, de alguien o de un modo de vivir diferente, distinto, que puede generar duda, miedo, inquietud. Esta presencia escapa a las explicaciones lógicas pero al mismo tiempo escapa a las explicaciones maravillosas. En la lógica del relato, de lo que la voz narrativa cuenta, irrumpe una alteridad que no es posible explicar porque al mismo tiempo pertenece y no a lo que el relato construye como realidad. Son autores que, a través de sus obras, rompen con el estatuto tradicional de lo real y abren la brecha de otras nociones y categorías literarias. Muchos de ellos fueron caracterizados por Rama (1966) como raros. Aquí, cien años de raros (Rama, 1966) busca romper con la crítica literaria del momento y evidenciar una línea de fuga existente en la literatura uruguaya: los raros; aquella literatura extraña, insólita, que se desprende de la construcción homogénea de lo que consideramos como realidad. Son literaturas que, si bien parten del estatuto realista, terminan rompiéndolo, fisurándolo, agujerean la manera de escribir y leer que se tenía y posibilitan nuevos edificios teóricos literarios, nuevas mentalidades, nuevas formas de ser. Una literatura que provoca, que golpea, que agita, que incomoda, que cuestiona. En términos de Benítez (2018): "literaturas que despiertan otras poéticas y políticas de la imaginación, sensiblemente más liberadas de las mímesis analógicas integradas en el interior de los cánones realistas, a las que, de algún modo, dichas creaciones contribuyen a criticar" (p. 9).

La dinámica que teníamos en el grupo de investigación era por demás "tradicional" respecto de lo que a estudios literarios se refiere. Cada integrante del grupo se dedicaba al estudio de un autor (Mario Levrero, Felisberto Hernández, Héctor Galmés, L. S. Garini, Juan Introini, Tarik Carson, por mencionar algunos ejemplos). Nos reuníamos asiduamente para discutir nuestros avances así como aquel corpus teórico que nos sostenía y participábamos de encuentros, congresos, simposios en los que compartíamos y nos animábamos a debatir nuestros estudios. Más allá de estos encuentros, se trataba de una investigación (como la mayoría de las literarias) en solitario, como de quien investiga frente a libros y su saber exclusivamente en el ámbito académico. Nada más. Sin embargo, parecía que estas literaturas en concreto necesitaban de otro tipo de investigación. Estas literaturas inquietantes propiciaron también un cuestionamiento en la forma de estudiarlas. Fueron ellas que nos impulsaron a salir de la rutina del escritorio, de la lectura y la escritura; nos empujaron a buscar otra forma de investigación literaria. Una investigación situada que contemplara a los receptores. Receptores concretos y particulares (no el lector ideal y abstracto que 
plantea Jauss en su Teoría de la Recepción). Era necesario crear un espacio para problematizar estas literaturas con otros lectores, muchos de ellos quizás ajenos a la academia pero con una interesantísima manera de interpretar que era (y es) imperativo escuchar e intercambiar. La extensión universitaria fue la respuesta.

En 2015 surgió la idea de crear el primer EFI en el ámbito de la Licenciatura de Letras, llamado "Taller abierto de lectura, interpretación y creación en torno a literaturas no realistas, insólitas y fantásticas en el Hospital Vilardebó". De la investigación a la extensión. El EFI está disponible a estudiantes de toda la FHCE así como a estudiantes de la Facultad de Psicología y Ciencias Sociales de la UdelaR. Su objetivo general es propiciar un espacio de actividad de debate en torno a los textos, de modo que las integrantes participen activamente mediante consideraciones, opiniones, interpretaciones y creaciones verbales y gráficas, "experimentando creaciones de imaginación artística, así como de cuestionamientos de cierta idea de realidad y/o de ficción" (Benítez, en Benítez y Pagano, 2017:27).

EI EFI apuesta a la integralidad más arriba conceptualizada y esta es percibida en sus tres momentos:

El primero que tiene que ver con la planificación, preparación y ejecución de talleres semanales de lectura e interpretación de textos uruguayos no realistas en el Espacio Psicosocial del Hospital Vilardebó ubicado en Montevideo. En este espacio, los estudiantes van en duplas conjuntamente con la pareja docente. Es el momento de llegada al campo y la organización del espacio para la actividad de lectura, cuando recorremos las salas para avisar a las usuarias sobre la actividad. Podríamos exponer que la función universitaria más presente en este momento es la extensión, ya que implica un contacto directo con la cotidianidad de las usuarias y a su vez el establecimiento de una instancia dialógica de producción de saber en un espacio colectivo.

En un segundo momento, en la facultad, en la que, por un lado, problematizamos y reflexionamos en torno a las vivencias que se tienen en los talleres, nos damos un espacio a la escucha de emociones y sensaciones para luego poder pensarnos alrededor de ellas. ¿Tengo prejuicios sobre personas que padecen de cierto diagnóstico psiquiátrico? ¿Cómo me siento en la recorrida de las salas, invitando a las personas a participar? ¿Cómo me siento en el taller: qué escucho, qué pienso, qué reflexiono? ¿Cómo me voy? ¿Cómo quedo luego de esa experiencia? Esta instancia es muy importante entre el grupo que se gesta debido a los intercambios que se realizan entre los propios estudiantes. Es fascinante observar las distintas perspectivas de acuerdo con la carrera que están estudiando y cómo estas repercuten en los sentires y en los comentarios que se hacen a partir de compartir lo que la experiencia genera. Por otro lado, existe una instancia más teórica en la que integrantes del grupo de investigación presentan los autores que estudian y los textos a debatir en el taller del hospital. También invitamos a psicólogos del hospital para trabajar en torno a la población del mismo y a integrantes de la Asamblea Instituyente (organización protagonista en la lucha por una nueva ley sobre la salud mental) para dialogar sobre la salud mental desde una perspectiva de derechos humanos así como acerca de la situación legal y la lucha social en el Uruguay y la región. En esta circunstancia prima la enseñanza, aunque también es cierto que los encuentros en la facultad son más que un espacio teórico, ya que la necesidad de compartir el transitar subjetivo del estudiante por el taller cobra 
singularidad y protagonismo. Entonces también es cuando se observa más la interdisciplina del EFI en tanto instancia de diálogo, de pensamiento colectivo, cada estudiante desde su historia como sujeto y desde su recorrido como tal.

Finalmente, un tercer momento, que podríamos pensar que es donde se percibe más la investigación, es cuando el estudiante elabora un trabajo final alrededor de una temática que le haya parecido fundamental y sea necesario detenerse, pensar y crear. Vale resaltar que cada estudiante tiene la posibilidad de llevar la referencia de alguno de los talleres. En general, se comienza desde una observación, luego esta se transforma en observación participante, y al cabo el estudiante deviene en coordinador al animarse a proponer algún texto, estudiarlo y leerlo en el taller así como formular preguntas a las participantes y encauzar el debate que se genere.

El EFI funciona todos los semestres pares. En 2015 llevamos a cabo la experiencia de los talleres de lectura tanto en el sector femenino como masculino del hospital. Ese año nos acompañaron estudiantes de la Licenciatura en Letras, Antropología, y la Tecnicatura en Corrección de Estilo. En 2016, por determinadas cuestiones de ajustes de horarios (parte del cuerpo docente del EFI lo realiza de forma honoraria, lo que demuestra la actual coyuntura de la situación de la extensión en la UdelaR), nos vimos obligados/as, desde el cuerpo docente, a priorizar la realización del taller en un sector solo, el femenino. Esta decisión no fue al azar. Al momento de tener que ajustarnos a un solo sector elegimos el femenino ya que este cuenta con muchas menos actividades de "rehabilitación" que el sector masculino, lo que refleja una clara situación de violencia de género que merece ser analizada: la mujer, en este caso, no solo es oprimida en tanto mujer sino también en tanto "loca". Observamos las condiciones de carencias existenciales de las mujeres que se encuentran internadas, su situación de exclusión, de rechazo, de despojo, de incomprensión, de pobreza, de falta de escucha afectiva. En estas ocasiones, asistieron estudiantes de la Licenciatura en Letras y Filosofía.

En 2017 contamos, asimismo, con estudiantes de las Licenciaturas en Educación, Lingüística, Letras, Antropología, Psicología, y la Tecnicatura en Corrección de Estilo. En 2018, la presencia fue de estudiantes de Psicología, Licenciatura en Letras, Antropología, Historia y Corrección de Estilo.

\section{Un EFI en un manicomio}

No por casualidad elegimos el Hospital Vilardebó para la realización del EFI. En aquel entonces (2015), y aun en la actualidad, la salud mental en el Uruguay estuvo en boga. Muchas organizaciones sociales se nuclearon para derribar la ley que hasta el año 2017 imperó: la № 9.581 llamada "Psicópata", que fue aprobada el 8 de agosto de 1936 (en plena dictadura de Gabriel Terra). Se trataba de una ley con una perspectiva psiquiátrica, biologicista, que apuntaba a la exclusión y al aislamiento social de los considerados "psicópatas". Pasada la década de 2010, diversas organizaciones sociales con perspectiva de derechos humanos se nuclearon en la Comisión por una Nueva Ley de Salud Mental y tomaron como ejemplo las leyes y políticas de salud mental de la región, como las de Brasil y Argentina, para luchar por la derogación de la Ley 9.581 y la creación de una ley de salud mental en términos de derechos humanos. La misma terminó por aprobarse el 24 de agosto de 2017, la Ley № 19.529, 
llamada "Salud Mental". Si bien no se logró incorporar todas las propuestas que las organizaciones sociales habían elaborado, esta nueva ley implicó un cierto avance. ${ }^{1}$

En este marco de lucha social, se crea el EFI que no solo acompaña la misma sino que además trata la salud mental desde la perspectiva de derechos humanos. Ejemplo de esto es la necesidad de cambiar ciertas nominaciones, como la de interno o paciente, por la de usuario/a de salud mental (Amarante, 2009), apostar al intercambio en el debate que los textos nos proponen así como promover espacios de escucha y encuentros horizontales entre la literatura, los docentes, los estudiantes y los que circunstancialmente se encuentran internados en el Hospital Vilardebó. En los talleres no nos interesa saber por qué están internados, cuál es su diagnóstico, sino el debate que surge entre todos y todas a partir de determinado texto literario no realista uruguayo.

Los talleres suceden dentro del hospital psiquiátrico, dentro de un manicomio (más allá de que la nueva ley prevé el cierre de instituciones asilares y monovalentes en los artículos 37 y 38). En términos de Goffman (1988), todo manicomio es una "institución total". Los mecanismos de toda institución total reside en aislar socialmente a los individuos internados por un período apreciable de tiempo y en la "tendencia absorbente" simbolizada por "los obstáculos que se oponen a la interacción social con el exterior" (p. 18). Todas las actividades básicas de los internos quedan reducidas a un mismo lugar y a la programación estricta de una misma autoridad. La prolongada estadía del interno tiene como efecto una "desculturación", "un 'desentrenamiento' que lo incapacita temporariamente para encarar ciertos aspectos de la vida diaria en el exterior, si es que vuelve a él y en el momento que lo haga" ( $p$. 26). El "quedar libre" va a depender del significado que le atribuya el interno y si es aceptado por la autoridad de la institución encarnada, por ejemplo, en el psiquiatra. Pero la paradoja está en lo que el autor denomina como efecto de looping, donde "el individuo comprueba que su respuesta defensiva falla en la nueva situación: no puede ya defenderse en la forma de costumbre, poniendo cierta distancia entre la situación mortificante y su yo" (p. 46). La "desculturación" y la imposibilidad de defenderse conllevan dicha heteronomía del interno. Estas instituciones promueven la pérdida de identidad y de autonomía de la persona así como una fuerte estigmatización y aislamiento por parte de la sociedad (Goffman, 1961), tal como se nos revela en el taller realizado en el Hospital Vilardebó, convirtiéndose este hospital de agudos en un hospital de crónicos (Benítez y Pagano, 2017:28).

Nuestra intervención extensionista, además de visualizar e interpretar la problemática del hospital psiquiátrico, tiene como fundamento proponer un modelo de transformación tanto a nivel de salud mental como de la investigación en literatura no realista.

\section{De la extensión a la investigación}

A partir de esta experiencia de extensión hemos comenzado a cuestionar las metodologías tradicionales en la investigación literaria. Como ya lo expresamos en otro apartado, históricamente la investigación literaria es una investigación en solitario: una persona que inves-

1) Recomendamos la lectura del texto "Incidencia de los marcos jurídicos en los cambios culturales: a propósito de la ley 19.529", de Gianella Bardazano, publicado en: https://www.hemisferioizquierdo.uy/single-post/2018/05/26/Incidencia-de-los-marcos-jur\%C3\%ADdicos-en-los-cambios-culturales-a-prop\%C3\%B3sito-de-la-ley-195291 
tiga determinada obra se nuclea principalmente con dicha obra y con la crítica que existe hasta el momento. Casi no se realizan entrevistas o grupos de discusión. El pensamiento colectivo queda relegado. Y mucho más la recepción de la obra. En primer lugar, porque la teoría de la recepción creó el concepto de lector ideal o "tipo" no situado, sin ser ubicado en determinado contexto histórico, temporal. En segundo lugar, porque mayoritariamente la literatura procura estudiar la obra "en sí" y no sus posibles derivaciones (como sería el caso de la recepción). Esta práctica de extensión provocó la ruptura de esta tradición y la creación de otra manera de investigar. Una investigación en colectivo y situada. Nos referimos a dar espacio a la recepción de las obras literarias y a su debida problematización y politización. La importancia de esta práctica de extensión para la investigación radica en la posibilidad de acceder a una fenomenología de recepción doblemente ajena a la academia (en el sentido de que no forma parte de ella y tampoco tiene contacto alguno con ella) y, por lo tanto, alejada del "libreto" que con frecuencia gravita sobre nosotros a la hora de aproximarnos a las obras literarias. La investigación se enriquece con el acceso a los talleres en la medida en que estos nos ponen en contacto con lectores "de carne y hueso", "de aquí y ahora", alejados de aquel lector ideal que tanto se le criticara a Jauss.

Los talleres constituyeron un espacio abierto de expresión, diálogo y comunicación con un sector social excluido y marginal que tiene el derecho de expresarse y contar sus historias. Se trata de producir con estas mujeres conocimientos acerca del efecto que genera la lectura de estas literaturas no realistas en ellas y en nosotros/as. Se trata de investigar literatura en colectivo, situada en determinado lugar y tiempo. Se trata de investigar en la extensión. Chidichimo expone:

"Si se reconoce que el tema central de la problemática de la extensión hoy es la producción de conocimiento sobre la realidad, esto implica ir más allá de situar acciones de transferencia al medio de lo producido dentro de la academia, ya que la idea de transferir algo implica una concepción del otro despojado, insuficiente, diferente, carente desde un nosotros que tiene algo para decir, aportar o enriquecer. Arribar a la idea de construcción del conocimiento entre diferentes instituciones, organizaciones y actores sociales y políticos tiene consecuencias en las formas que asumirá el diálogo con estos otros. Se constituye así una forma de relación de la universidad con su sociedad a la cual considera no solo como objeto de la acción sino como fuente de saber". (2017:332)

Las usuarias, participantes del taller, dejan de ser enfermas psiquiátricas, dejan de ser internas de un hospital psiquiátrico, dejan de ser las otras despojadas para ser, juntamente con los estudiantes y el cuerpo docente, un nosotros que lee, se pregunta, debate, intercambia, responde, crea saber. El saber literario que se produce es un saber integral, que deviene del intercambio de distintos sujetos, todos fundamentales.

Podemos decir que toda experiencia de extensión busca transformar entendiendo a la transformación como proceso de transmutación. Este horizonte no siempre es audible y visible. Algunas experiencias son criticadas porque en ellas se percibe más asistencialismo y menos proceso de transformación por parte de los actores universitarios y la sociedad. Creemos que no es lo que sucede con esta experiencia. La misma no solo rompe los supuestos básicos de una investigación literaria tradicional al crear otras posibilidades en estos estudios sino que también transforma al estudiante que participa, al cuerpo docente, y se 
establece en el hospital como una línea de fuga, una práctica desmanicomializante dentro de una lógica manicomial. Vayamos por partes:

En cuanto a los estudiantes, varias son las transformaciones que se perciben. Grato es recordar expresiones como: "Siento que, gracias a esta práctica, no soy la misma". Los estudiantes que comienzan no son los mismos cuando finalizan. Apreciamos un proceso en el que van problematizando sus miedos, sus prejuicios y preconceptos acerca de las personas que padecen cierto diagnóstico psiquiátrico. Al principio se los puede observar temerosos, tímidos, pero luego, a través de los encuentros en la facultad y de las participaciones en el taller, van quitando de sí mismos los vendajes y los ropajes de preconceptos, prejuicios y miedos. Comienzan a descubrir otra forma de ver la locura, las psicosis. Una perspectiva que considera a las psicosis como un discurso y no como un trastorno de la personalidad sujeto a patrones estadísticos de normalidad funcionales a la lógica neoliberal, cuyo discurso de realidad se rige por mantener un statu quo. Comienzan a empoderarse $\mathrm{y}$, al igual que las usuarias, participan, intervienen, preguntan y hasta coordinan un taller. En los encuentros en la facultad se los ve movilizados, motivados y comprometidos con la práctica: "Para mí esta experiencia va más allá de los créditos que puedo obtener, es un compromiso ético y político ante la sociedad".

Por otro lado, cada estudiante aporta desde su perspectiva, esto es, no solo desde su subjetividad en tanto sujeto con historia particular y singular sino también desde su disciplina. Los saberes de cada especificidad se comparten, se intercambian, se nutren uno del otro. Así, por ejemplo, los estudiantes de Psicología en su mayoría se concentran en pensar cómo la literatura no realista favorece a la rehabilitación de la usuaria; los de Ciencias de la Educación, en el proceso de aprendizaje en condiciones manicomiales y marginales, en lo atinente a desarrollar una práctica educativa al margen de la sociedad y de las condiciones educativas que todo proceso de aprendizaje espera tener. Los estudiantes de la Licenciatura en Letras se concentran en los debates que se generan en torno a las lecturas de los cuentos; los de Filosofía, en la relación de la teoría filosófica que trabaja conceptos como locura o encierro con la práctica concreta de la experiencia. Estas problematizaciones o cuestionamientos que comparten van acoplándose con los otros y así las reflexiones pasan a ser macro y micro al mismo tiempo. La interdisciplina favorece la posibilidad de apreciar lo micro transversalizado por una mirada global, en la que dejan de existir comportamientos estanco del saber para pasar a considerar el conocimiento de forma compleja en tanto particularidades comprendidas y unidas en cierta totalidad.

Con relación al cuerpo docente, la primera transformación vivida fue el hecho de romper con la lógica tradicional del saber magistral o vertical. Esta práctica nos obliga, felizmente, a poner en práctica la educación emancipadora de la que habla Paulo Freire (1984), alejándonos de la bancaria. Esta práctica implica un aprendizaje no solo para estudiantes y usuarias sino para el cuerpo docente. ¿Acaso no debería ser así en todas las prácticas educativas que llevamos adelante? En esta experiencia la horizontalidad es la protagonista. Los docentes dejamos de portar determinado saber entendido como el "verdadero" porque todos los actores tienen un saber que es necesario compartir e intercambiar. Apenas tenemos el rol de referencia en los talleres, un rol móvil, que puede ser tomado no solo por estudiantes sino también por las propias usuarias. Algunas de ellas proponen textos literarios, su lectura y su posible dinamización. El recuerdo de esta práctica en las otras funciones universitarias 
es fundamental para poder mantener la ruptura de la lógica del saber magistral y habilitar así prácticas educativas emancipadoras. En otros términos, la forma en que desarrollamos dicha práctica de extensión atraviesa las fronteras e impregna a las funciones de enseñanza e investigación. Al dar aulas en la facultad recordamos estas prácticas y proponemos otras formas de estar y ser en clase (no solo desde, por ejemplo, intentar sentarnos en círculo, sino también asignar momentos de coordinación de la clase para todos los participantes, asegurar espacios de debate e intercambio, entre otros). Del mismo modo sucede en la investigación. Como hemos expresado, dicha experiencia permitió transformar la manera de estudiar literatura: quebrar con la soledad del investigador para dar apertura a instancias colectivas desde la recepción concreta y singular de estas literaturas.

Esta experiencia también impacta en el hospital. No sin resistencias, se ha convertido en una pausa o paréntesis a la lógica manicomial. Se trata de un espacio desmanicomializante dentro de una lógica manicomial. ¿Por qué decimos "no sin resistencias"? Porque todos los años tenemos que vencer las barreras burocráticas del hospital para que nos permitan desarrollar la práctica y porque, una vez permitida, hemos recibido resistencia por parte de algunos funcionarios que allí trabajan. "Ese espacio no es bueno para ella, la altera"; "ella no puede ir, no está en condiciones". ¿Por qué "la altera"? ¿Porque tiene un espacio para poder hablar y ser realmente escuchada? ¿Por qué no puede ir si se trata de un espacio libre, es decir, que no se necesita el permiso del médico tratante para asistir? No debemos olvidar que se trata de una institución que busca mantener y justificar un modelo monovalente (a pesar de los artículos aprobados en la nueva ley persiste, en gran parte de la sociedad, la resistencia al cierre de dichas instituciones), basado en un lógica manicomial que justifica prácticas de estigmatización, heteronomía y de sujeción, que cronifica la situación de las personas que se encuentran internadas y no privilegia la "rehabilitación". Tal como mencionamos, consideramos las psicosis como una forma particular de articulación narrativa singular de sufrimiento de un sujeto. De ahí que en psicoanálisis se parte de una concepción dinámica del sujeto, en permanente conflicto, y no de una concepción estática o estadísticamente normal. Esta concepción de sujeto implica para nosotros un posicionamiento ético de encuentro con una manera particular de narrar el sufrimiento dentro de una trama social. Considerar las psicosis como un discurso, como fenómeno particular de articulación de lenguaje, es sostener que ahí hay un decir y hay algo para ser escuchado, que existe una historia que se anuda con la vida personal y cotidiana de un sujeto en sociedad. $Y$ esto puede promoverse con los talleres realizados en el hospital.

Consideramos que todas las personas que asisten al taller tienen un saber para compartir. Allí se les da un espacio de escucha, de contención. Se rescata la palabra como canal de comunicación, de encuentro, de unión. No se trata de considerarlas como enfermas psiquiátricas sino como personas que participan de un taller, cuya presencia es importante, que tienen un saber para aportar necesario y fundamental para el desarrollo del espacio. Un ejemplo de los saberes que aportan se relaciona con el lenguaje.

Tanto las críticas freudiana y lacaniana como la tradición estructuralista -y la convergencia de ambas corrientes en las ideas desconstruccionistas - han señalado la relación entre lenguaje y psicosis. Al respecto, Todorov dice: "Si la psicosis en general es una perturbación en la relación entre el yo y la realidad exterior, entonces el discurso psicótico será un discurso que fracasa en su trabajo de evocación de esta realidad; en otras palabras, en su 
trabajo de referencia" ${ }^{2}$ (1980:103). Por su parte, los textos no realistas presentan un proceso lingüístico sui generis vinculado, también, a su tratamiento de la referencialidad. El lenguaje se presenta, de este modo, como gran eje articulador entre las psicosis, el discurso y las literaturas fantásticas a través de su función referencial:

"Lo fantástico supone (...) el desajuste entre el referente literario y el lingüístico (pragmático), es decir, la discordancia entre el mundo representado en el texto y el mundo conocido (...) el narrador se ve obligado a combinar de forma insólita nombres y adjetivos para intensificar la capacidad de sugerencia. Podemos decir, entonces, que de connotación reemplaza a la denotación". (Roas, 2001:28-29).

A este respecto, nos parecen elocuentes algunas observaciones correspondientes al día de la lectura de "El árbol", un cuento de de Juan Introini, que trata de una visita del protagonista a la casa de una amiga que tiene un gran árbol en el centro de su jardín. En la visitas suceden eventos extraños entre el protagonista, la amiga y el árbol. Le Cercle (1994) llama "sugestiones onomatopoéticas" a los fenómenos en los que la resonancia fonológica de ciertas palabras dispara asociaciones de campos semánticos distintos de los del contexto pero que comparten dicha palabra. Por su parte, Vegh (1995) menciona las confusiones producto de homofonía como comportamiento característico del discurso psicótico:

Luana $^{3}$ : -Pero una figura... para un árbol genealógico, es la figura paterna... (min. 24)

Adelina: -Ella cortó de raíz para que crecieran las cosas buenas. (min. 34)

En los ejemplos anteriores se observa el fenómeno al que aludíamos más arriba: el cambio de una palabra hacia otro contexto disparado por cierta autorreferencialidad al momento de la recepción. El árbol del cuento —en su sentido literal—es resignificado por Luana hacia una de sus acepciones figuradas; similar operación hace Adelina con respecto a las raíces. Opera en los ejemplos previos una dislocación del referente con respecto al discurso, esto es, en términos saussureanos, un cambio del sistema de signos a los que se opone el signo en cuestión (en este caso, la palabra árbol). Se corrobora, de este modo, el fracaso en la función referencial en torno al que Todorov (1980) desarrolla su caracterización del discurso psicótico. Las interpretaciones de las usuarias van en la línea del lenguaje figurado, negando el elemento referencial de los textos.

Lo mismo sucede con ciertas lecturas autorreferenciales que realizan de los textos. Muchas de ellas se ubican en el lugar de la alteridad, de encontrarse del otro lado de la sociedad. Curioso, al ser este uno de los ejes fundamentales de las literaturas fantásticas. ${ }^{4}$ Son elocuentes, en este sentido, las palabras de Jackson: "Desde un mundo racional, 'monológico', la alteridad solo puede ser conocida y representada como lo extranjero, lo irracional, '/o loco', 'lo malo'” (2001:143, cursivas nuestras). Esa percepción de marginalidad en cuanto a cierta noción de normalidad fue captada y expresada con frecuencia por las usuarias; y cabe preguntarse si, al margen de la fuerte autorreferencialidad que caracterizan sus lecturas, no hay un autorreconocimiento pertinente, un reconocimiento de la propia marginalidad, no solo

2) La traducción es nuestra.

3) Todos los nombres de las usuarias son ficcionalizados para proteger su identidad.

4) Así lo indica Todorov (1982), quien clasifica las temáticas fantásticas en "temas del yo" y "temas del tú". 
con respecto a una sociedad sino a un discurso: "Ella lo que no quería era ser un ser social", repite Adelina el día de la lectura de "Felicidad", cuento de María Inés Silva Vila, narrado en primera persona por una joven mujer que relata parte de su vida hasta la actualidad. De una síntesis de su pasado vuelve a la actualidad describiendo su trabajo: estar sentada frente a una vidriera mientras le hacen distintos peinados. "Ella era contravectaria", dice Lucía, revertoria, recurriendo a bellos neologismos que acaso quisieran capturar el sentido de controversial, rebelde (contra-el-vector). Estamos frente a una lectura marginal de una literatura de-lo-marginal. Se resalta el esfuerzo por rescatar un grado de referencialidad de los textos. El fracaso en la función de referencialidad se troca en esfuerzo por rescatarla a la hora de interpretar.

Por último, los talleres en el hospital ofician, para muchas usuarias, como un espacio de alfabetización a través del arte, en este caso, de la literatura. Conjuntamente, conocemos autores uruguayos, practicamos la lectura a través de los cuentos, aprendemos el significado de palabras nuevas, debatimos sobre temáticas en las que no estábamos familiarizadas y ejercitamos la escritura o aprovechamos para retomarla.

La integralidad es uno de los resultados de esta práctica, no solo en lo que respecta a las funciones universitarias, sino con relación a los saberes entre estudiantes de diversas carreras, en tanto promueven la interdisciplina, usuarias y docentes, de vivencias y de historias. Todo ello propicia una transformación subjetiva en cualquier persona que participa de la experiencia.

\section{A modo de cierre}

Esta experiencia de extensión bajo el dispositivo de un EFI constituyó (y esperemos continúe constituyendo), por un lado, instancias de encuentro de trabajo con la palabra, estableciéndose como espacio de lazo social que genera transformaciones. El miedo y la angustia ante lo desconocido son transmutados por sentimientos afectivos, el respeto y el intercambio horizontal al compartir un espacio de debate, incertidumbres, inquietudes, creatividad, alegría y tristeza. La transformación de las mujeres en el Hospital Vilardebó a través de la escucha de los relatos fantásticos conlleva a una transformación subjetiva, donde devienen "en lectoras críticas evidenciando otra recepción de estas literaturas no realistas" (Benítez y Pagano, 2017:29). Se trata de alteridad otra, ya no despojada de la escucha de su singularidad y su historia, sino de una alteridad que a través del arte y de la palabra se historiza de otra manera en el lenguaje. Como propuesta instituyente, los talleres consistieron en expresión y creación cultural, en la apertura de un espacio de "alfabetización a través del arte" que permitió "historizarse y poder nombrarse" (Benítez y Pagano, 2017:29) por medio de la palabra. En términos de Hounie: "Cuando las formas de la palabra se ofrecen a bordear lo que no es palabra, acontece la creación como posibilidad" (2016:175). Consiste en proponer una transformación que es la transmutación espacio-temporal de las lógicas manicomiales mediante el arte, la escucha horizontal, el despliegue de lo grupal y la pausa o el paréntesis manicomial que hace a una práctica desmanicomializante (Benítez y Pagano, 2017:30).

Por otro lado, esta experiencia es la evidencia de cierta laguna teórica en las investigaciones de las literaturas no realistas. Creemos que esa laguna puede deberse a la falta de un estudio de recepción situado y concreto de esas literaturas, donde pueda problematizarse el modo de investigación "tradicional" solitario, ya que los talleres son un camino valioso para 
poder abordar teóricamente estos textos mediante otra práctica, de carácter colectivo y oral. Detectar esta evidencia fue posible gracias al intercambio e interpretaciones generadas en los talleres de lectura y debate entre docentes, estudiantes y usuarios y usuarias de salud mental. Es decir, creemos insuficiente estudiar estos textos de manera "tradicional", tanto en su complejidad poética como en su potencial crítico. También estos textos fueron clave en cuanto a la generación de un lazo social con las usuarias del hospital, sobre todo por su crítica de una visión racional de lo real en el mundo.

Varios son los desafíos a los que esta práctica nos enfrenta. Por una parte, los desafíos institucionales: sortear la barrera burocrática del hospital año a año para garantir el funcionamiento de la experiencia así como intentar tender puentes con aquellos funcionarios que ejercen resistencia al desarrollo de la misma, acercarnos, conversar con ellos, invitarlos a participar. Por otra parte, los desafíos políticos; a saber: democratizar y extender el desarrollo de estas prácticas en el marco de la nueva Ley de Salud Mental, que no sea solo papel, que se observe en el accionar del día a día, y exigir menos recortes y más recursos para la universidad, para que la extensión universitaria tenga más presupuesto para el desarrollo de actividades como estas. No olvidemos que el cuerpo docente de esta experiencia es honorario. ¿El desarrollo de prácticas como esta debe quedar librado a la disponibilidad y compromiso de las personas? En coyunturas políticas actuales, debemos más que nunca, resistir, proponer y crear.

\section{Referencias bibliográficas}

Amarante, P. (2009). Superar el manicomio: Salud mental y atención psicosocial. Buenos Aires: Topia.

Arocena, R. (2011). Curricularización de la extensión: ¿por qué, cuál, cómo? En Arocena, R.; Tomassino, H.; Rodriguez, N.; Sutz, J.; Álvarez Perdosian, E.; Romano, A. (Eds.). Cuadernos de Extensión. Integralidad. Tensiones y Perspectivas (pp. 9-19). Montevideo: Comisión Sectorial de Extensión y Actividades en el Medio (CSEAM).

Bardazano, G. (mayo de 2018). Incidencia de los marcos jurídicos en los cambios culturales: a propósito de la ley 19.529. Hemisferio Izquierdo. Recuperado de: https://www.hemisferioizquierdo.uy/single-post/2018/05/26/Incidencia-de-los-marcos-jur\%C3\%ADdicos-en-los-cambios-culturales-a-prop\%C3\%B3sito-de-la-ley-195291

Benítez, H. y Pagano, E. (2017). Transformaciones a partir del Espacio de Formación Integral: "Taller abierto de lectura, interpretación y creación en torno a literaturas no realistas, insólitas y fantásticas" en el Hospital Vilardebó. Conexão,14(1), 25-31. Recuperado de: http://www.revistas2.uepg.br/index.php/conexao/article/ view/10587/6262

Benitez, H. (2018). Mímesis en otro lado: disrupciones uruguayas. En Benitez, H. (Ed.). El otro lado: disrupciones en la mimesis Lo insólito, lo fantástico y otros desplazamientos en la narrativa uruguaya (desde los años sesenta a las primeras décadas del siglo XXI) (pp. 7-15). Montevideo: Ediciones Universitarias, Unidad de Comunicación de la Universidad de la República (UCUR).

Cano Menoni, A. (2015). Integralidad en la universidad de la república. Apuntes para el debate universitario. En Cruz, P.; Fernández, V.; Lorieto, A.; Repetto, L.; Vienni, B.; Von Sanden, C. (Eds.). En_clave Inter 2014. Educación superior e interdisciplina. Seminario En_clave Inter del Espacio Interdisciplinario (pp. 111-119). Montevideo: Espacio Interdisciplinario de la Universidad de la República.

— (2017). La extensión universitaria y la Universidad Latinoamericana: hacia un nuevo "orden de antici- 
pación" a 100 años de la revuelta estudiantil de Córdoba. Revista +E versión en línea, 7(7), 6-23. Santa Fe, Argentina: Ediciones UNL.

Castillo, J.; Correa, N.H.; Parentelli, V. y Romero, M. (2017). Espacios de Formación Integral: transformaciones y desafíos para la enseñanza y la docencia de Ciencias Económicas y Administración. Revista +E versión en línea, 7(7), 156-163. Santa Fe, Argentina: Ediciones UNL.

Chidichimo, M. (2017). Derribando muros: un aporte desde la extensión universitaria a los procesos de sustitución del manicomio. Revista +E versión en línea, 7(7), 330-337. Santa Fe, Argentina: Ediciones UNL.

Freire, P. (1984). Pedagogía del Oprimido. Madrid: Siglo Veintiuno.

Goffman, E. (1961). Estigma. La identidad deteriorada. Buenos Aires: Amorrortu.

(1988). Internados. Ensayos sobre la situación social de los enfermos mentales. Buenos Aires: Amorrortu. Jackson, R. (2001). Lo oculto de la cultura. En Roas, D. (Ed.). Teorías de lo Fantástico (pp. 141-153). Madrid: Arco/Libros.

Hounie, A. (2016). El dolor tiene muchas caras... La subjetividad comprometida: fragmentos para una estética del dolor. En Hounie, A.L.; Fernández Caraballo, A.M. (Eds.). Políticas del dolor. La subjetividad comprometida. Un abordaje interdisciplinario de la problemática del dolor (pp. 170-179). Montevideo: Ediciones Universitarias, Unidad de Comunicación de la Universidad de la República (UCUR).

Le Cercle, J. (1994). Phylosophy of Nonsense. London: Routledge.

Menéndez, G. (2017). Resignificación de la extensión a 100 años de la Reforma Universitaria de 1918. Revista +E versión en línea, 7(7), 24-37. Santa Fe, Argentina: Ediciones UNL.

Rama, A. (1966). Aquí cien años de raros. Montevideo: Arca.

Scarciófolo, S.M. (2017). Marta Samatan. Historia de una mujer reformista. Santa Fe, Argentina: Ediciones UNL.

(junio de 2018). Marta Elena Samatan. Historia de una mujer reformista. Maestra, abogada, extensionista, escritora, reformista. Hemisferio Izquierdo. Recuperado de: https://www.hemisferioizquierdo.uy/ single-post/2018/06/20/\%E2\%80\%9CMarta-Elena-Samat\%C3\%A1n-Historia-de-una-mujer-reformista\%E2\%80\%9D-Maestra-abogada-extensionista-escritora-reformista

Roas, D. (2001). La amenaza de lo fantástico. En Roas, D. (Ed.). Teorías de lo Fantástico (pp. 7- 44). Madrid: Arco/Libros.

Todorov, T. (1980). Os gêneros do discurso. São Paulo: Martins Fontes.

(1982). Introducción a la literatura fantástica. Barcelona: Buenos Aires S.A.

Tommasino, H.; Cano, A.; Castro, D.; Santos, C. y Stevenazzi, F. (2010). De la extensión a las prácticas integrales. En Rectorado, La extensión en la transformación de la enseñanza: los Espacios de Formación Integral (s/p). Montevideo: UdelaR.

Vegh, I. (1995). Una cita con la psicosis. Rosario: Homo Sapiens. 